\title{
Transient gels in colloid-polymer mixtures studied with fluorescence confocal scanning laser microscopy
}

\author{
Nynke A.M. Verhaegh ${ }^{\mathrm{a}, *}$ Daniela Asnaghi ${ }^{\mathrm{b}}$, Henk N.W. Lekkerkerker ${ }^{\mathrm{a}}$ \\ a Van 't Hoff Laboratory, Debye Institute, Utrecht University, Padualaan 8, 3584 CH Utrecht, \\ The Netherlands \\ ${ }^{\mathrm{b}}$ Department of Physics and INFM, University of Milan, Via Celoria 16, 20133 Milan, Italy
}

Received 17 July 1997; received in revised form 27 August 1998

\begin{abstract}
We study the structure and the time evolution of transient gels formed in colloid-polymer mixtures, by means of fluorescence Confocal Scanning Laser Microscopy (CSLM). This technique is used in conjunction with novel colloidal silica particles containing a fluorescent core. The confocal micrographs reveal that there exist large differences in the local structure within a single system. At a given time there are regions where the gel structure consists of alternating patterns of colloid-rich and colloid-poor regions with a characteristic length scale and regions where the gel structure becomes disrupted by the formation of fractures. The number of fractures increases with time. It is speculated that the increase of the number of fractures leads to a weakening of the strength of the gel such that it eventually collapses under gravity. (c) 1999 Elsevier Science B.V. All rights reserved.
\end{abstract}

\section{Introduction}

It is well known that by adding non-adsorbing polymers to a colloidal suspension an attraction between colloidal particles may be induced, giving rise to phase separation. This attraction is called depletion-induced and was first described by Asakura and Oosawa [1] and Vrij [2]. Colloids are surrounded by a depletion zone from which the polymers are sterically excluded. When the colloids are close enough their depletion zones overlap, causing an unbalanced osmotic force. This results in an attractive interaction between the colloidal particles, the range and depth of which can be tuned by

* Corresponding author. 
the diameter $\left(\sigma_{\mathrm{p}}\right)$ and the volume fraction $\left(\phi_{\mathrm{p}}\right)$ of the polymer, giving rise to different types of phase diagrams [3-8].

The phase separation for the case of a relatively short range of attraction $\left(\sigma_{\mathrm{p}} / \sigma_{\mathrm{c}}=\right.$ 0.08 , where $\sigma_{\mathrm{c}}$ is the diameter of the colloid) has been studied extensively [9-11]. If there is enough polymer present, the system undergoes a fluid-crystal phase separation. For larger amounts of polymer, a variety of non-equilibrium behaviour is encountered, namely nucleation-like and spinodal-like aggregation, and transient gelation (i.e. the gels have a finite lifetime). These non-equilibrium processes are characterized by the presence of an intense ring in the scattering pattern. The ring, which in nucleation-like aggregation appears only after a latency time, collapses in all cases towards zero angle. However, in transient gelation its time evolution is arrested during the gel life time. Indications have been given that these transient gels are made of fractal aggregates filling all the available space. In a recent experiment transient gels in a colloid-polymer mixture with a larger range of attraction $\left(\sigma_{\mathrm{p}} / \sigma_{\mathrm{c}}=0.25\right)$ have been investigated [12]. A stationary ring in the scattered intensity pattern is again observed during the gel life time. The scattering profiles show the Porod decay $\left(I(q) \sim q^{-4}\right)$ at high $q$, thus indicating the presence of sharp interfaces. Both the presence of a ring at non-zero angle and the Porod decay are characteristic for phase separating binary liquid mixtures, and have been observed in colloid-polymer mixtures with a broad attraction range $\left(\sigma_{\mathrm{p}} / \sigma_{\mathrm{c}}=1\right)$ during fluid-fluid phase separation [8]. This suggests that, for the case of the intermediate range attraction $\left(\sigma_{\mathrm{p}} / \sigma_{\mathrm{c}}=0.25\right)$, the initial phase separation process gets arrested by gelation [13].

The polymer-induced particle gels are transient, in contrast with the permanent particle gels that arise in irreversibly aggregating systems $[14,15]$. This is presumably due to the fact that the energy of polymer-induced bonds is in the order of the particles' thermal energy. It has been argued that the reversible nature of the bonds allows a rearrangement of the gel structure, eventually leading to the collapse of the gel [912]. Computer simulations on colloidal aggregation have indeed established that in the case of finite attractive interactions, restructuring occurs leading to local compactification [16-19]. So far, experimental information on the structural rearrangement leading to the collapse of transient gels is limited. In our previous work [12] the small angle light scattering profiles showed a slow increase in intensity at low wavevector during the gel lifetime, indicating a loss of order in the system. We have tentatively ascribed this small angle behaviour to large scale rearrangement in the structure.

In the present work we investigate the restructuring of transient gels by means of fluorescence Confocal Scanning Laser Microscopy (CSLM) [20]. This powerful technique allows a direct visualization of the structure, both in situ and time-resolved [21-25]. Here, it has been used in conjunction with novel silica particles containing a fluorescent core [22-25]. The confocal micrographs show that the gel structure which initially is rather regular, becomes locally distorted by the formation of fractures. The number of fractures in the gel increases with time until the gel becomes disconnected and collapses. 
The paper is organized as follows. In Section 2 we introduce the system and the experimental techniques. In Section 3 we present the experimental results. The data are discussed in Section 4 which also contains the conclusions.

\section{Experimental}

\subsection{Materials}

The silica particles were nucleated in a micro emulsion following the method of Osseo-Asare and Arriagada [26,27]. Using the procedures described by Van Blaaderen et al. [28] these nuclei, with a diameter of $42 \mathrm{~nm}$, were first covered with a silica layer (thickness of $26 \mathrm{~nm}$ ) in which the fluorescent dye fluorescein isothiocyanate (FITC, Isomer I, Sigma) was incorporated. Then they were grown with a pure silica coating to a final diameter of $230 \mathrm{~nm}$ as was determined with Static Light Scattering and Dynamic Light Scattering. The polydispersity (standard deviation divided by mean radius) equals $4.1 \%$ and the particle density $1.69 \mathrm{~g} / \mathrm{ml}$. Finally, the particles were coated with 1-octadecanol (stearylalcohol, Merck) to provide a steric stabilization [29]. The particles were dispersed in cyclohexane (Janssen), where their refractive index is matched. In cyclohexane the particles have a (almost) hard-sphere interaction [30].

Two samples of polydimethylsiloxane (PDMS, Janssen) with different gyration radii were used. The molecular weight $\left(M_{\mathrm{w}}\right)$ and the radius of gyration $\left(r_{\mathrm{g}}\right)$ were determined with Static Light Scattering (Zimm plots) and with Gel Permeation Chromatography. For the smaller polymer we found $M_{\mathrm{w}}=9.7 \times 10^{3} \mathrm{~g} / \mathrm{mol}$ and $r_{\mathrm{g}}=14 \mathrm{~nm}$, while for the larger polymer $M_{\mathrm{w}}=2.11 \times 10^{5} \mathrm{~g} / \mathrm{mol}$ and $r_{\mathrm{g}}=23 \mathrm{~nm}$.

\subsection{Colloid-polymer mixtures}

Colloids and polymers were suspended in cyclohexane at various volume fractions, the polymer volume fraction $\phi_{\mathrm{p}}$ being defined as the polymer number density times a spherical volume with radius equal to the polymer gyration radius. In the following we shall refer to mixtures with the small polymer as mixtures $\mathrm{A}\left(\sigma_{\mathrm{p}} / \sigma_{\mathrm{c}}=0.12\right)$, and to mixtures with the large polymer as mixtures $\mathrm{B}\left(\sigma_{\mathrm{p}} / \sigma_{\mathrm{c}}=0.20\right)$. For mixtures $\mathrm{A}$ the colloid volume fraction $\phi_{\mathrm{c}}$ was in the range between $1 \%$ and $13.5 \%$, and $\phi_{\mathrm{p}}$ between $8 \%$ and $125 \%$. For mixtures $\mathrm{B} \phi_{\mathrm{c}}$ ranged between $3 \%$ and $13 \%$, and $\phi_{\mathrm{p}}$ between $10 \%$ and $150 \%$. The mixtures were prepared in glass tubes where their phase behaviour could be studied visually. Homogenization by fierce shaking brought a sample back to the beginning of its phase evolution.

The systems undergoing transient gelation were transferred into flat windows glass capillaries (Vitro Dynamics: various optical paths, ranging from 0.2 to $1 \mathrm{~mm}$; width $=4$ $\mathrm{mm}$; height $=5 \mathrm{~cm}$ ) which were sealed off by melting. Homogenization of the mixtures in these capillaries was rather difficult. However, even with the thinnest capillaries we used, a rather good reproducibility of the observed processes was obtained. 


\subsection{Fluorescence confocal scanning laser microscopy}

In fluorescence CSLM the specimen is probed, point by point, with a diffraction limited spot of laser light, coupled by a lens to a point detector. Thus, only fluorescence from this very small area is detected, ensuring depth discrimination and enhanced lateral resolution. Micrographs are constructed by digital data storage and scanning, and are therefore also suitable for data processing.

The colloid-polymer mixtures were studied with a Bio-Rad MRC-600 Confocal Microscope mounted on a Zeiss Axioplan equipped with an argon-ion laser. A filter block was installed, containing a dichroic reflector, an excitation filter and a detection filter, such that the excitation wavelength was $488 \mathrm{~nm}$ and fluorescence above $515 \mathrm{~nm}$ was detected. The intensity of the laser beam at $488 \mathrm{~nm}$ was about $5 \mathrm{~mW}$. Since the dye is concentrated in the core of the particles, only a small fraction of laser light is absorbed before reaching the focal point. We used a $40 \times$ oil immersion lens with a numerical aperture of 1.3 , such that the lateral resolution was $200 \mathrm{~nm}$ and the axial resolution $650 \mathrm{~nm}$. In the system used in this work the size ratio of the colloidal particles and their fluorescent cores inhibits a visual distinction between touching particles, so that we only observe differences in number density.

The capillary $(0.2 \mathrm{~mm}$ optical path) was positioned vertically and sections of the transient gel parallel to the direction of gravity were visualized. Confocal images of $512 \times 512$ pixels were obtained in a scanning time of $1 \mathrm{~s}$. With the applied magnification these images covered an area of $237 \times 237 \mu \mathrm{m}^{2}$. The evolution of the structures in the transient gel was displayed on a television screen in 'live-mode' and recorded on a video tape. During a measurement, 8-bit images of the gel structure at approximately $35 \mu \mathrm{m}$ behind the glass wall were grabbed with a delay time of ten seconds, for a period extending beyond the gel lifetime.

\section{Results}

\subsection{Phase behaviour}

The phase behaviour of the colloid-polymer mixtures A and B was studied as a function of the colloid and polymer volume fractions by visual inspection. In addition a laser beam was shined onto the capillaries and the speckles fluctuations were observed on a projection screen. We did not observe significant differences in phase behaviour for the two mixtures.

Basically, we could distinguish among three scenarios as a function of the polymer concentration. (i) At low $\phi_{\mathrm{p}}$ the mixtures are thermodynamically stable, they do not phase separate but slowly settle (over weeks) into a sediment that is crystalline at the top. (ii) At higher $\phi_{\mathrm{p}}$ the mixtures rapidly phase separate (within an hour) into a dense amorphous bottom phase and a dilute upper phase. In the upper phase small crystallites become visible after days. These crystallites subsequently settle forming a thin layer 
$(\approx 1 \mathrm{~mm})$ on top of the amorphous lower phase. Without discounting the possibility that crystallization also occurs in the bulk, we found with dark field microscopy (Carl Zeiss microscope) that nuclei originate at the glass wall. From those nuclei which were properly positioned with respect to the direction of view, the bright (blue and green) Bragg reflections were observed. Similar wall-induced crystallization has been reported long ago by Kose and Hachisu [31] and more recently by Kaplan et al. [32]. (iii) At even higher $\phi_{\mathrm{p}}$ the dense lower phase only separates after a latency time. During this latency time no fluctuating speckles in the scattering pattern are observed, indicating that the system is in a gel state. After the latency time the gel collapses into a dense, amorphous lower phase by a rapid descend of an interface, leaving behind a very dilute upper phase. The typical latency time for the systems studied here, as determined by visual observation, turned out to be about $300 \mathrm{~s}$. No signs of iridescence are observed over several weeks.

\subsection{Fluorescence confocal scanning laser microscopy}

With fluorescence CSLM, we studied the systems at colloid and polymer compositions where transient gelation took place. After homogenization, it took at least $30 \mathrm{~s}$ to position the sample in the microscope. By this time, the gel had already formed. Therefore, confocal microscopy images were observed during the lifetime and the collapse of the gel. The micrographs shown are representative for the evolution of the structure in the transient gel, as seen in the live-mode on a television screen. No significant differences were observed for mixtures $\mathrm{A}$ and $\mathrm{B}$.

In Fig. 1 we present four confocal micrographs of a mixture $A$ with $\phi_{\mathrm{c}}=12 \%$ and $\phi_{\mathrm{p}}=61 \%\left(c_{\mathrm{p}}=8.55 \mathrm{mg} / \mathrm{ml}\right)$ during the lifetime of the gel approximately $30 \mathrm{~s}(\mathrm{a}), 60 \mathrm{~s}$ (b), $120 \mathrm{~s}$ (c) and $240 \mathrm{~s}$ after homogenization. We observe textured images consisting of bright and dark domains, corresponding to colloid-rich and colloid-poor regions. The gel structure exhibits a dominant length scale which is associated with a typical domain size. By comparing Fig. 1a-d we see that during the gel lifetime this dominant length scale is maintained. Although the overall impression of the images does not change, local rearrangement in the pattern of dark and bright domains can be observed if we carefully focus on a part of the structure in the sequence of micrographs. Approximately $270 \mathrm{~s}$ after homogenization, a collective flow of the gel structure was observed along the direction of gravity. This agrees with the observation that the collapse of the gel is associated with a rapid descend of an interface separating the colloid-rich lower phase and colloid-poor upper phase.

In order to obtain a characteristic length scale of the structures displayed in Fig. 1 we have performed the 2-dimensional Fast Fourier Transform (FFT) of the micrographs using the IDL [33] program, and computed the power spectra as the square modulus of the FFT. Because of the relatively large size of the scanned image compared to the 'field of view' of the lens, the images were nonuniformly illuminated. Therefore we had to process the images to perform the FFT. The illumination profile was obtained by iteratively smoothening the image of a regular gel structure by means of standard 

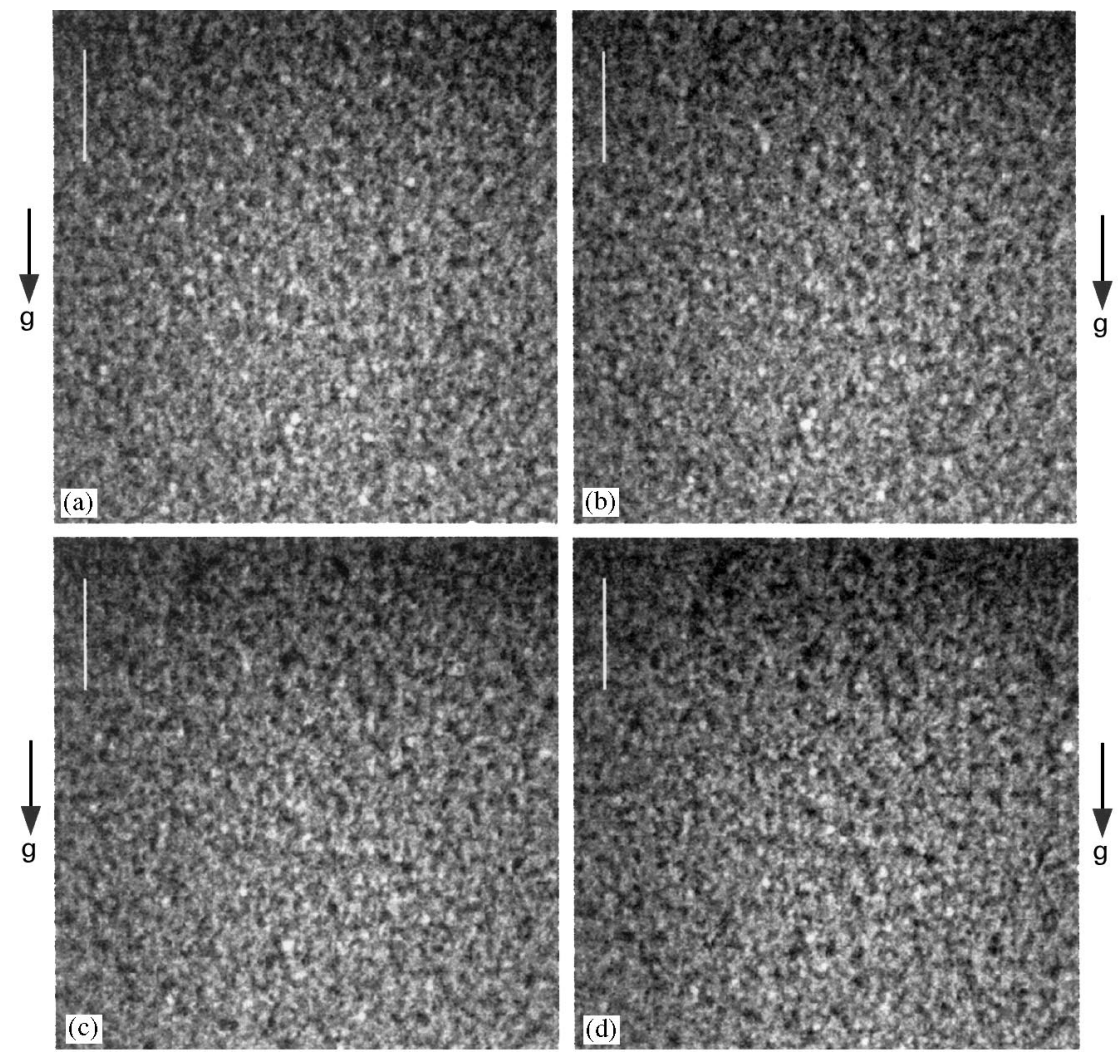

Fig. 1. CSLM micrographs of mixture A with $\phi_{\mathrm{c}}=12.1 \%$ and $\phi_{\mathrm{p}}=61.0 \%\left(c_{\mathrm{p}}=8.55 \mathrm{mg} / \mathrm{ml}\right)$ during the gel lifetime: (a) approximately $30 \mathrm{~s}$, (b) $60 \mathrm{~s}$, (c) $120 \mathrm{~s}$ and (d) $240 \mathrm{~s}$ after homogenization. The bar equals $50 \mu \mathrm{m}$. The arrow with g indicates the direction of gravity. The micrographs have been smoothened and the contrast has been enhanced to provide a clearer image.

techniques (box car average [33]). The gray values in the micrographs were subsequently divided by this profile. A Hanning filter [33] was used to correct for the finite size of the picture. Finally, the mean gray value was subtracted from the data before performing the FFT, in order to consider just density fluctuations. In Fig. 2 the square modulus of the spatial Fourier transforms is plotted, for the data of Fig. 1. Initially a bright ring at a non-zero frequency is observed (Fig. 2a). By radially averaging the square moduli of the FFT we found that the peak is centered at a frequency of 15 pixels. Taking into account that an array of 512 pixels measures $237 \mu \mathrm{m}$ in real space, this corresponds to a wavevector $q=3.9 \times 10^{3} \mathrm{~cm}^{-1}$. The corresponding length scale in real space is given by $L=2 \pi / q=16 \mu \mathrm{m}$. This is in good agreement with previous experiments on a closely related system [12]. As time elapses, the ring becomes elongated in the horizontal direction, indicating that the gel structure in real space exhibits anisotropy, presumably due to gravity. This anisotropy is hard to be discerned in the original micrographs. 

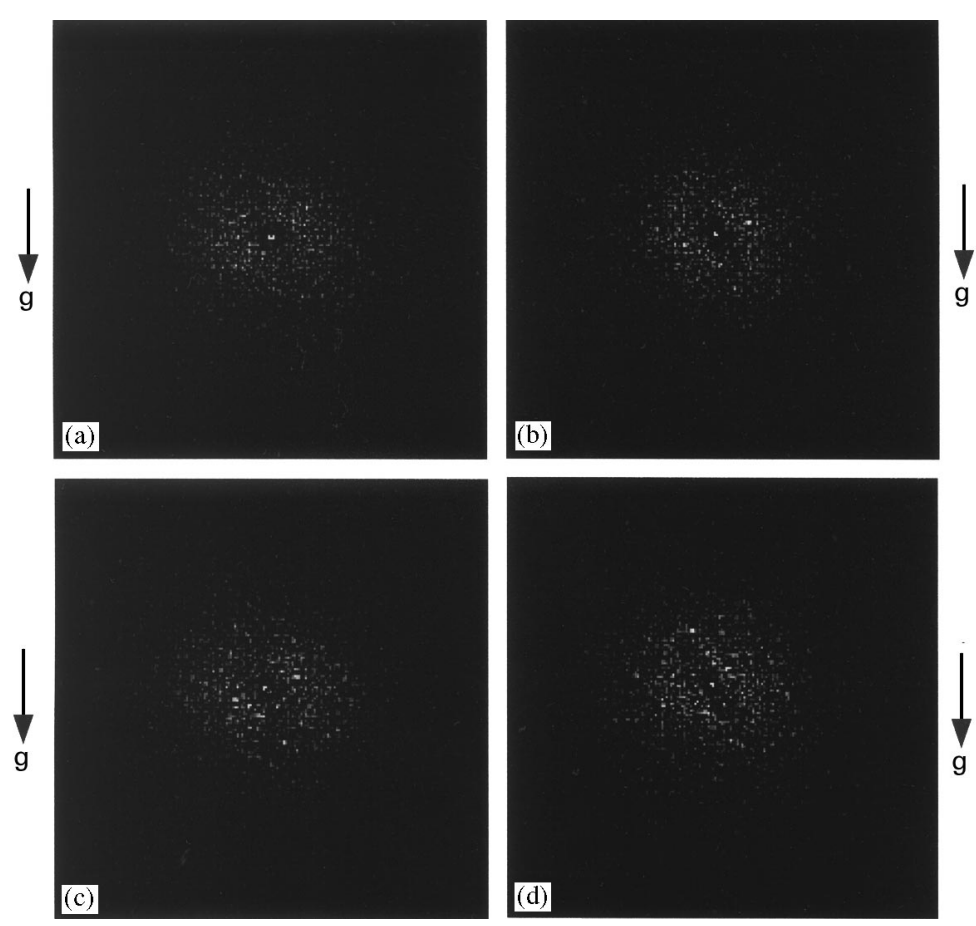

Fig. 2. Square modulus of spatial Fourier transforms of the micrographs in Fig. 1. (a) $30 \mathrm{~s}$ after homogenization, (b) $60 \mathrm{~s}$, (c) $120 \mathrm{~s}$, (d) $240 \mathrm{~s}$. The arrow with g indicates the direction of gravity. For convenience we only show the central portion $(256 \times 256$ pixels $)$ of the $512 \times 512$ array in frequency space. The images are sharpened for a better visibility.

In Fig. 3 the time-evolution ((a) $30 \mathrm{~s}$, (b) $60 \mathrm{~s}$, (c) $100 \mathrm{~s}$ and (d) $120 \mathrm{~s}$ after homogenization) of the gel structure is shown for mixture B with $\phi_{\mathrm{c}}=12 \%$ and $\phi_{\mathrm{p}}=96 \%\left(c_{\mathrm{p}}=6.60 \mathrm{mg} / \mathrm{ml}\right)$. Fig. 3a shows a regular structure consisting of dark and bright domains which resembles the gel structure in Fig. 1. On the bottom of the micrographs, the structure remains relatively undisturbed. However, on the top a fracture is seen to develop (Fig. 3b-d). These fractures grow mostly in the direction of gravity. Parcels of the gelled system (several times the typical domain size) become disconnected from the network. In a number of cases these parcels diffuse randomly through these cracks, whereas in other cases, they have been seen to move in a direction opposite to gravity.

In Fig. 4 ((a) $230 \mathrm{~s}$, (b) $240 \mathrm{~s}$, (c) $250 \mathrm{~s}$ and (d) $260 \mathrm{~s}$ after homogenization) we illustrate another strongly distorted gel structure during the late stage of the gel lifetime in mixture B $\left(\phi_{\mathrm{c}}=12 \%, \phi_{\mathrm{p}}=123 \%, c_{\mathrm{p}}=8.52 \mathrm{mg} / \mathrm{ml}\right)$. Some fractures change in shape and size by displacement of fragments of the gel structure, whereas other fractures maintain their shape and size from Fig. 4a-d. We observed that large fragments of the gel structure move via translations and rotations through the fractures in a random fashion. 

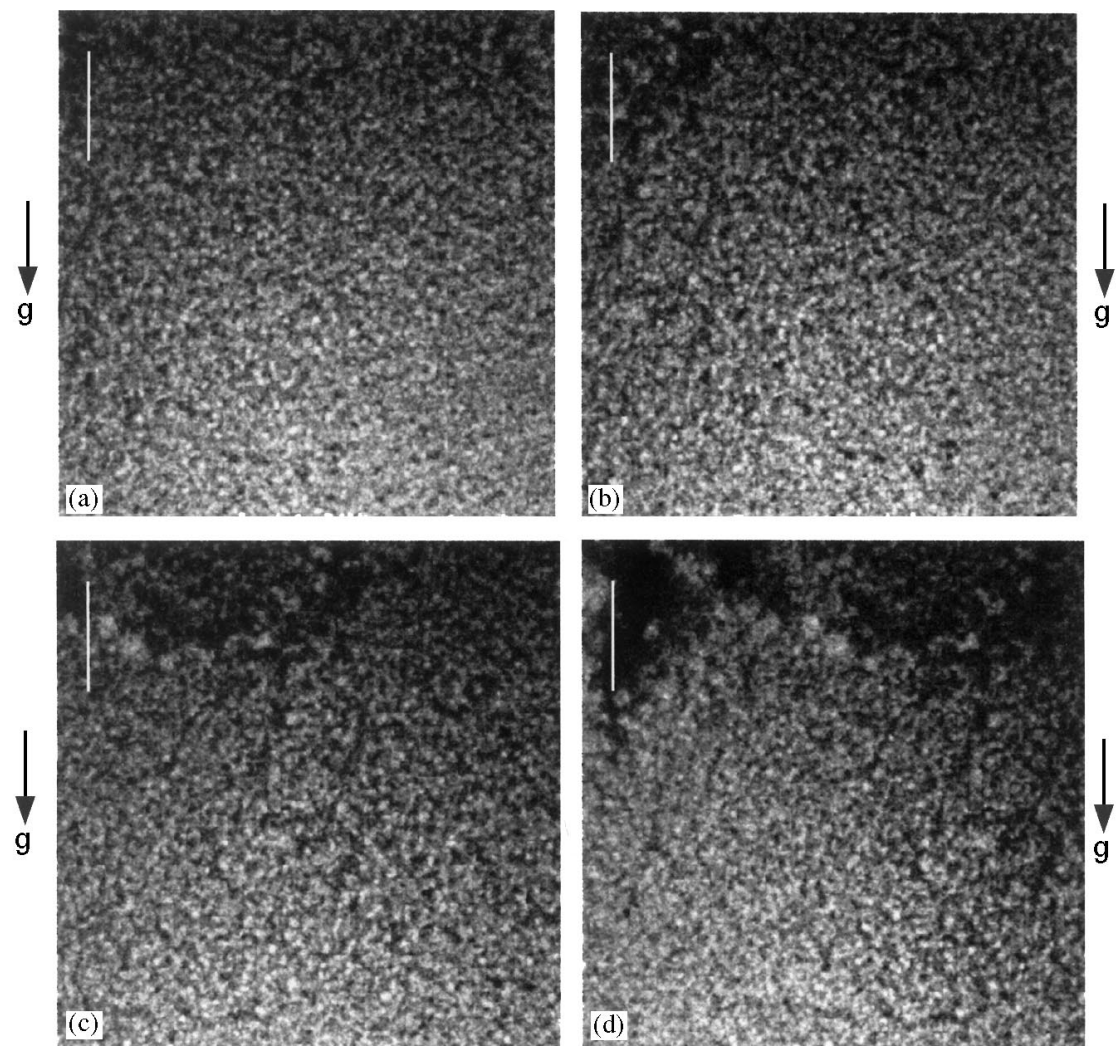

Fig. 3. CSLM micrographs of mixture B with $\phi_{\mathrm{c}}=11.6 \%$ and $\phi_{\mathrm{p}}=95.7 \%\left(c_{\mathrm{p}}=6.60 \mathrm{mg} / \mathrm{ml}\right)$ during the gel lifetime: (a) approximately $30 \mathrm{~s}$, (b) $60 \mathrm{~s}$, (c) $100 \mathrm{~s}$ and (d) $120 \mathrm{~s}$ after homogenization. The bar equals 50 $\mu \mathrm{m}$. The arrow with $\mathrm{g}$ indicates the direction of gravity.

\section{Discussion and conclusions}

From our confocal micrographs it is evident that the structure of the gel continually rearranges over all length scales during its lifetime (see Fig. 1), i.e. it is a "dynamic" gel. This is due to the reversible nature of the depletion induced bonds. Simulations have shown that this continuous rearrangement leads to compactification of the clusters that make up the gel [16-19].

A striking observation that we obtained by studying transient gels with the fluorescence CSLM was the fact that there exist substantial differences in the local structure within a single system at the same time. During the gel lifetime there are regions where the structure consists of an alternating pattern of colloid-rich and colloid-poor regions with a characteristic length scale. Simultaneously, the gel contains regions which are disrupted by the formation of fractures. From the videotape recording the evolution of the system we observed that the number of fractures increases with time. Although difficult to discern from the micrographs by eye the regular structures are characterized 

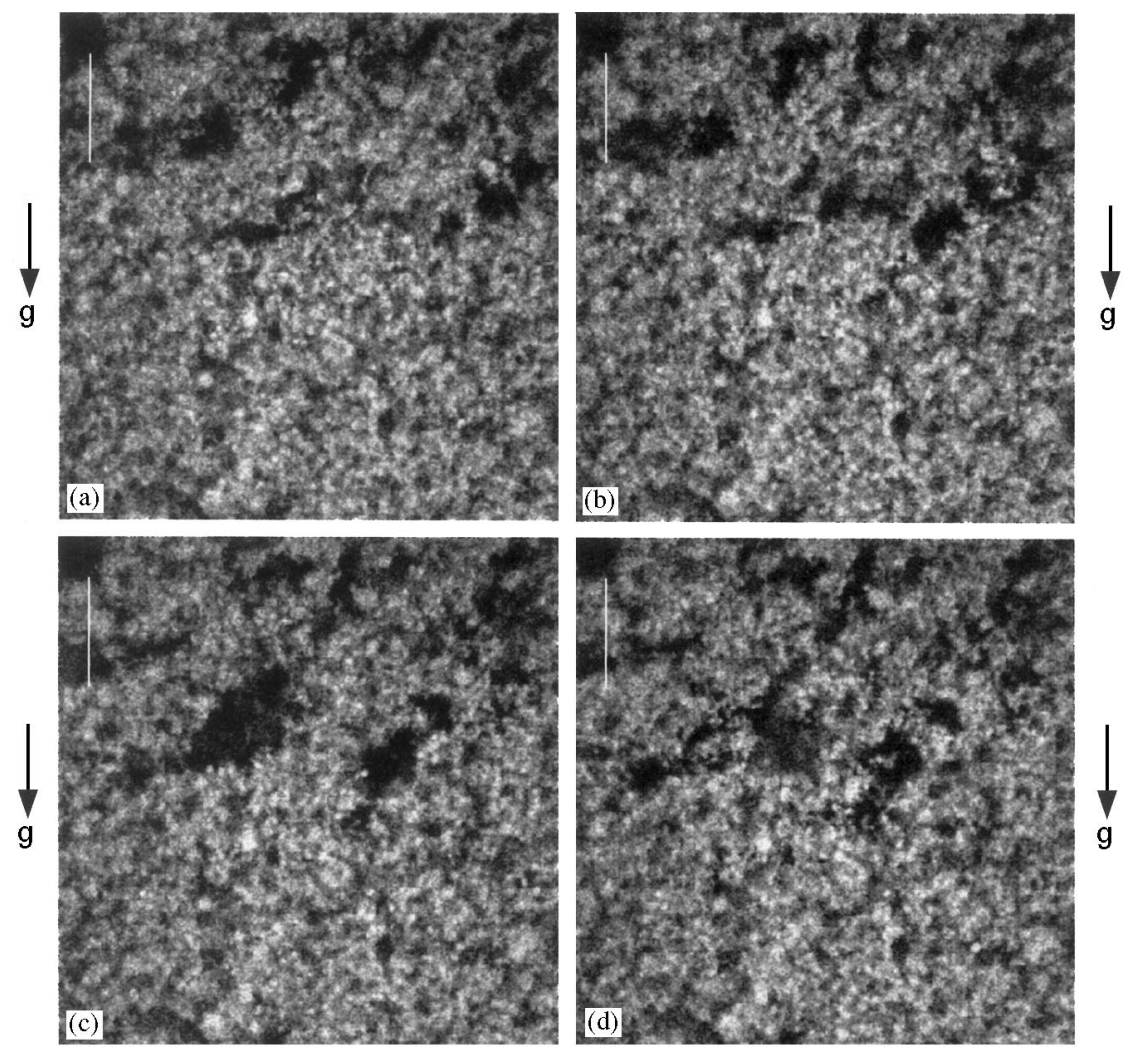

Fig. 4. CSLM micrographs of mixture B with $\phi_{\mathrm{c}}=12.5 \%$ and $\phi_{\mathrm{p}}=123 \%\left(c_{\mathrm{p}}=8.52 \mathrm{mg} / \mathrm{ml}\right)$ during the gel lifetime: (a) approximately $230 \mathrm{~s}$, (b) $240 \mathrm{~s}$, (c) $250 \mathrm{~s}$ and (d) $260 \mathrm{~s}$ after homogenization. The bar equals $50 \mu \mathrm{m}$. The arrow with $\mathrm{g}$ indicates the direction of gravity.

by a growth in anisotropy, as is revealed by the power spectra. This is probably an effect of gravity.

We speculate that the end of the lifetime of the gel is connected to the increase of the number of fractures which weaken the gel strength to such an extent that it collapses under gravity. Similarly, Allain et al. [34] have shown that for the settling of a gel one can distinguish between a slow settling regime when the gelled suspension settles collectively and a fast settling regime when the gel is disrupted by fractures along which upward solvent flow can be observed.

It is interesting to relate our CSLM data with small angle light scattering observations during the lifetime of the gel. In a light scattering experiment the intensity profile results from an average over the scattering volume. Based on our CSLM data, we expect that the scattering volume consists of both regular structures and structures distorted by fractures. The regular structures give rise to a ring in the intensity profile while the large scale distorted structures are expected to scatter strongly in the forward direction. Since the number of fractures increases with time we expect that the forward scattering 
increases with time. This is exactly what is observed [12]. We conclude that confocal microscopy and light scattering yield complementary information on the restructuring process.

\section{Acknowledgements}

This work was supported by the Stichting voor Fundamenteel Onderzoek der Materie (Foundation for Fundamental Research on Matter) which is part of the Nederlandse Organisatie voor Wetenschappelijk Onderzoek (Netherlands Organization for the Advancement of Research) and the Agenzia Spaziale Italiana. The collaboration between the Milan and Utrecht groups was made possible by the financial support of the European Community for the Network Colloid Physics. We thank Willem Hage (Netherlands Institute for Developmental Biology, Utrecht) for his support with the CSLM measurements, and Alfons van Blaaderen for several illuminating discussions of this work.

\section{References}

[1] S. Asakura, F. Oosawa, J. Chem. Phys. 22 (1954) 1255.

[2] A. Vrij, Pure Appl. Chem. 48 (1976) 471.

[3] A.P. Gast, C.K. Hall, W.B. Russel, J. Colloid Interface Sci. 96 (1983) 251.

[4] H.N.W. Lekkerkerker, W.C.K. Poon, P.N. Pusey, A. Stroobants, P.B. Warren, Europhys. Lett. 20 (1992) 559.

[5] W.C.K. Poon, J.S. Selfe, M.B. Robertson, S.M. Ilett, A.D. Pirie, P.N. Pusey, J. Phys. II France 3 (1993) 1075.

[6] S.M. Ilett, A. Orrock, W.C.K. Poon, P.N. Pusey, Phys. Rev. E 51 (1995) 1344.

[7] F. Leal Calderon, J. Bibette, J. Bias, Europhys. Lett. 23 (1993) 653.

[8] N.A.M. Verhaegh, J.S. Van Duijneveldt, J.K.G. Dhont, H.N.W. Lekkerkerker, Physica A 230 (1996) 409.

[9] P.N. Pusey, A.D. Pirie, W.C.K. Poon, Physica A 201 (1993) 322.

[10] W.C.K. Poon, A.D. Pirie, P.N. Pusey, Faraday Disc. 101 (1995) 65.

[11] W.C.K. Poon, A.D. Pirie, M.D. Haw, P.N. Pusey, Physica A 235 (1996) 105.

[12] N.A.M. Verhaegh, D. Asnaghi, H.N.W. Lekkerkerker, M. Giglio, L. Cipelletti, Physica A, accepted for publication.

[13] F. Sciortino, R. Bansil, H.E. Stanley, P. Alstrom, Phys. Rev. E 47 (1993) 4615.

[14] M. Carpineti, M. Gilgio, Phys. Rev. Lett. 68 (1992) 3327.

[15] G. Dietler, C. Aubert, D.S. Cannell, P. Wiltzius, Phys. Rev. Lett. 57 (1986) 3117.

[16] W.Y. Shih, I.A. Aksay, R. Kikuchi, Phys. Rev. A 36 (1987) 5015.

[17] W.Y. Shih, J. Liu, W.H. Shih, I.A. Aksay, J. Stat. Phys. 62 (1991) 961.

[18] M.D. Haw, M. Sievwright, W.C.K. Poon, P.N. Pusey, Adv. Colloid Interface Sci. 62 (1995) 1.

[19] M.T.A. Bos, J. Van Opheusden, Phys. Rev. E 53 (1996) 5044.

[20] T. Wilson, Confocal Microscopy, Academic Press, London, 1990.

[21] L.G.B. Bremer, B.H. Bijsterbosch, P. Walstra, T. Van Vliet, Adv. Colloid Interface Sci. 46 (1993) 117.

[22] A. Van Blaaderen, Adv. Mater. 5 (1993) 52.

[23] A. Van Blaaderen, A. Imhof, W. Hage, A. Vrij, Langmuir 8 (1992) 1514.

[24] N.A.M. Verhaegh, A. Van Blaaderen, Langmuir 10 (1994) 1427.

[25] N.A.M. Verhaegh, J.S. Van Duijneveldt, A. Van Blaaderen, H.N.W. Lekkerkerker, J. Chem. Phys. 102 (1995) 1416.

[26] K. Osseo-Asare, F.J. Arriagada, Colloid Surf. 50 (1990) 321. 
[27] F.J. Arriagada, K. Osseo-Asare, Colloid Surf. 69 (1992) 105.

[28] A. Van Blaaderen, A. Imhof, N.A.M. Verhaegh, to be published.

[29] A.K. Van Helden, J.W. Jansen, A. Vrij, J. Colloid Interface Sci. 77 (1980) 418.

[30] C.G. De Kruif, W.J. Briels, R.P. May, A. Vrij, Langmuir 4 (1988) 668.

[31] A. Kose, S. Hachisu, J. Colloid Interface Sci. 55 (1976) 487.

[32] P.D. Kaplan, J.L. Rouke, A.G. Yodh, D.J. Pine, Phys. Rev. Lett. 72 (1994) 582.

[33] IDL, Interactive Data Language, Version 4, a product of Research System Inc., Boulder, Colorado, 1995. Direct inquiries to info@rsinc.com.

[34] C. Allain, M. Cloitre, M. Wafra, Phys. Rev. Lett. 74 (1995) 1478. 